\title{
BILAN DES INTRODUCTIONS DE SALMONIDÉS DANS LES LACS ET RUISSEAUX D'ALTITUDE DES HAUTES-PYRÉNÉES.
}

\author{
M. DELACOSTE (1) (2), P. BARAN (1), J.M. LASCAUX (1), \\ N. ABAD (2), J.P. BESSON (3).
}

\begin{abstract}
(1) Laboratoire d'Ingénierie Agronomique, Equipe Environnement Aquatique et Aquaculture, Ecole Nationale Supérieure Agronomique de Toulouse, 145 avenue de Muret, 31076 Toulouse Cedex, France.

(2) Fédération Départementale des A.A.P.P.M.A. des Hautes-Pyrénées, 20 boulevard du 8 mai 1945, 65000 Tarbes, France.

(3) Parc National des Pyrénées, 59 route de Tarbes, 65000 Tarbes, France.
\end{abstract}

\section{RÉSUMÉ}

Les introductions de Salmonidés ont été importantes au cours des 60 dernières années dans les lacs et ruisseaux d'altitude des Hautes-Pyrénées. Six espèces de Salmonidés ont été introduites dans des milieux qui, pour la plupart, étaient vierges de populations piscicoles : la truite commune (Salmo trutta L.), la truite arc-en-ciel (Oncorhynchus mykiss Walbaum), l'omble de fontaine (Salvelinus fontinalis Mitchill), l'omble chevalier (Salvelinus alpinus L.), le cristivomer (Salvelinus namaycush Walbaum) et le splake (Salvelinus fontinalis $x$ Salvelinus namaycush). Dans de très nombreux cas, ces introductions ont abouti à des acclimatations. En revanche, les naturalisations sont beaucoup plus rares. Seules les espèces lacustres (cristivomer et omble chevalier) se sont naturalisées dans la majorité des lacs où elles ont été introduites. Les conditions de reproduction constituent le facteur clé permettant d'expliquer la naturalisation des espèces. En ruisseau, il faut y ajouter la compétition avec l'espèce indigène (la truite commune), la pression halieutique ainsi que les conditions hivernales très rigoureuses. Les incidences écologiques des introductions sur les populations de truites communes indigènes sont faibles. En revanche, elles ne sont pas négligeables pour les populations de batraciens. Cette politique d'introduction a largement participé au développement de l'halieutisme dans ces milieux d'altitude. En cela, les introductions ont parfaitement répondu aux objectifs halieutiques qu'on leur avait fixés. L'acquisition de connaissances sur l'ensemble de la chaîne pyrénéenne constitue aujourd'hui une étape incontournable pour une politique de gestion globale des introductions.

Mots-clés : Salmonidae, truite commune, truite arc-en-ciel, omble de fontaine, omble chevalier, cristivomer, Salmo trutta, Oncorhynchus mykiss, Salvelinus fontinalis, Salvelinus alpinus, Salvelinus namaycush, introductions, lacs.

\section{EVALUATION OF SALMONID INTRODUCTIONS IN HIGH-ELEVATION LAKES AND STREAMS OF THE HAUTES-PYRENEES REGION.}

\begin{abstract}
Salmonid introductions have been numerous during the last 60 years in high-elevation streams and lakes of the Hautes-Pyrenees region. Six salmonid species : brown trout (Salmo trutta L.), rainbow trout (Oncorhynchus mykiss Walbaum), brook trout (Salvelinus fontinalis
\end{abstract}


Mitchill), arctic charr (Salvelinus alpinus L.), lake trout (Salvelinus namaycush Walbaum), and splake (Salvelinus fontinalis $x$ Salvelinus namaycush) have been introduced in these regions where originally no fish was present. Acclimatization was observed in many cases, but investigations revealed that few populations reproduce. Only lake species (lake trout and arctic charr) reproduce in most lakes into which they were introduced. Adequate spawning conditions were the most important feature required for salmonid naturalisation. But in streams, interspecific competition with brown trout, the effects of angling, and severe winter conditions also interfered. The ecological effects of these introductions on the native brown trout species were very weak. Consequences were more important for amphibian populations, many of which declined dramatically. Salmonid introductions allowed the development of sport fishing in these high-elevation regions. From this point of view, they perfectly answered their aims. Broad knowledge on the whole Pyrenean mountain range is now required for a global management policy of introductions.

Key-words : Salmonidae, brown trout, rainbow trout, brook trout, arctic charr, lake trout, Salmo trutta, Oncorhynchus mykiss, Salvelinus fontinalis, Salvelinus alpinus, Salvelinus namaycush, introductions, lakes.

\section{INTRODUCTION}

Les Salmonidés ont fait l'objet de très nombreuses tentatives d'introductions à travers le monde (MCCRIMMON, 1971 ; MCDOWALL, 1984 ; BAGLINIĖRE, 1991). C'est très souvent l'intérêt halieutique qui a prévalu à ces introductions (CROWL et al., 1992). FAUSCH (1988) considère que moins de $50 \%$ ont abouti à des naturalisations. Elles ont permis de constituer de nombreuses combinaisons d'espèces ( 78 combinaisons potentielles pour le continent nord-américain (FAUSCH, 1988)). Les incidences biologiques de ces introductions peuvent être importantes. La compétition interspécifique peut aboutir à des réductions d'effectifs, soit pour les espèces indigènes de la même famille (COOPER, 1970 ; NYMAN, 1970 ; MOYLE, 1976 ; WATERS, 1983), soit pour les espèces d'autres familles (CROWL et al., 1992). De plus, les conséquences génétiques des réductions de taille des populations indigènes ne sont pas négligeables en réduisant la taille du pool génique et en augmentant les risques de dérive génétique (KRUEGER et MAY, 1991). De même, la compétition et la prédation peuvent entraîner des diminutions importantes des populations d'amphibiens. RESETARITS (1995) observe une survie et une croissance nettement plus faibles des salamandres (Gyrinophilus porphyrictius) en présence d'ombles de fontaine (Salvelinus fontinalis). Dans les Pyrénées, différents témoignages indiquent la présence de truites communes dans certains lacs au cours du XIXème siècle. Ainsi, MASSAS (1859) signale les lacs d'Oô et de Clarabide comme étant " les plus féconds en truites " ou encore MOREAU (1863), qui signale l'activité de 2 guides de pêche " conduisant les touristes à la pêche à la mouche sur les lacs de montagne ". Les introductions de Salmonidés ont été nombreuses au cours de ce siècle. Elles ont très souvent été effectuées par les autorités responsables de la gestion de la pêche dans des milieux d'altitude qui n'étaient pas naturellement peuplés de poissons, mais également par des pêcheurs professionnels liés à I'hôtellerie touristique. Dans les Hautes-Pyrénées, aux environs de 1935, les trois quarts de ces lacs étaient encore vierges, et une soixantaine seulement étaient peuplés de Salmonidés (CHIMITS, 1960). La création de piscicultures par l'administration des eaux et forêts en 1936 a conduit à des programmes de peuplements systématiques, dans le but de développer le loisir pêche (CHIMITS, 1960). Ces programmes d'alevinage ont été poursuivis par la Fédération de pêche des HautesPyrénées. Les poissons sont introduits au stade alevin de 6 mois. Certaines espèces se sont naturalisées dans des sites géographiques bien précis (par exemple la truite arc-en-ciel dans le vallon d'Estibère, Hautes-Pyrénées (ELLIOTT, 1973 ; DUMAS, 1976)) avec des conséquences écologiques réduites pour l'espèce indigène (BARAN et al., 1995a). Mais les informations restent parcellaires pour cette zone géographique.

Le but de cet article est de dresser un bilan des introductions de Salmonidés effectuées dans les lacs et ruisseaux d'altitude du département des Hautes-Pyrénées, de déterminer les réussites et les échecs enregistrés, et de tenter de fournir des éléments explicatifs. Les conséquences de ces introductions seront également abordées, ainsi que des éléments guides pour la gestion. 


\section{SITES D'ÉTUDE}

Figure 1

Situation géographique.
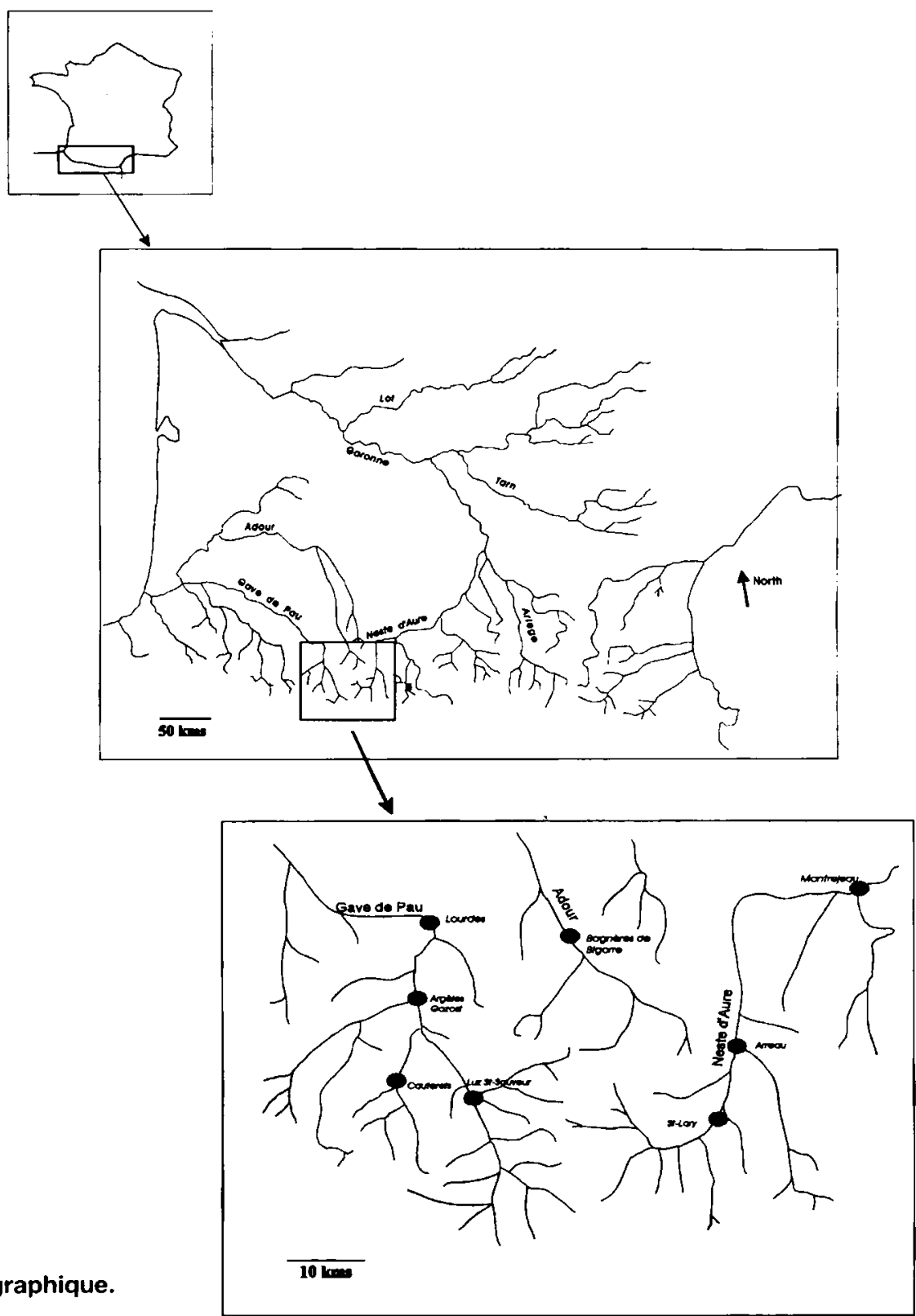

Figure 1

\section{Geographical situation.}

Le département des Hautes-Pyrénées compte 330 lacs et plus de $150 \mathrm{~km}$ de ruisseaux à des altitudes supérieures à $1500 \mathrm{~m}$. Les lacs, dont la très grande majorité sont d'origine naturelle, ont une surface comprise entre 0.1 et 120 ha. Les ruisseaux sont caractérisés par des régimes nivaux et nivaux-glaciaires selon l'altitude des sources (BARAN, 1995). Leur largeur varie, à $1500 \mathrm{~m}$, de 0.5 à $12 \mathrm{~m}$. Un nombre important d'entre eux sont court-circuités par des installations hydroélectriques.

Ces milieux sont caractérisés par des conditions environnementales rigoureuses, particulièrement en hiver (LAVANDIER, 1979). Les lacs sont totalement pris en glace de mi-novembre à mi-juin pour les plus bas en altitude, et de mi-septembre à août pour les plus élevés. En ruisseau, les prises en glace peuvent être totales ou partielles et les températures ne dépassent pas $2{ }^{\circ} \mathrm{C}$ pendant l'hiver (ELLIOTT, 1973 ; LAVANDIER, 1979). 


\section{MATÉRIELS ET MÉTHODES}

De 1985 à 1994, à l'initiative de la Fédération départementale de pêche et de pisciculture des Hautes-Pyrénées ainsi que du Parc National des Pyrénées, de nombreuses études ont été conduites sur les lacs et sur quelques ruisseaux. Ces études avaient pour objectif de dresser un bilan des populations piscicoles de ces milieux. Les pêches ont été réalisées sur plusieurs campagnes, à partir d'une batterie standard de filets monofilaments (PLASSEREAU, 1987, 1989 ; DAUBA, 1990, 1991). Dans les ruisseaux, les inventaires ont été réalisés par pêche électrique (matériel de type Martin-Pêcheur Dream Electronic, BARAN et al., 1995a, 1995b). A chaque échantillonnage, des écailles ont été prélevées sur 20 à 30 individus afin de réaliser une étude scalimétrique. Des rétro-mesures ont permis de déterminer la croissance des différentes espèces de poissons. Les croissances ont été ajustées selon le modèle de Von Bertalanffy. Un suivi halieutique, à partir de carnets de pêche, a permis d'estimer la capturabilité des différentes espèces dans des milieux où la population en place était connue (nombre de prises/pêcheur/heure).

\section{RÉSULTATS}

Six espèces de Salmonidés ont été introduites dans les lacs et ruisseaux d'altitude des Hautes-Pyrénées. II s'agit de la truite commune (Salmo trutta L.), de la truite arc-en-ciel (Oncorhynchus mykiss Walbaum), de l'omble de fontaine (Salvelinus fontinalis Mitchill), de l'omble chevalier (Salvelinus alpinus L.), du cristivomer (Salvelinus namaycush Walbaum), et du splake (Salvelinus fontinalis $x$ Salvelinus namaycush). Les dates de premières introductions, le nombre de lacs et de ruisseaux où l'espèce fut introduite, où elle est acclimatée actuellement, et ceux où elle est naturalisée sont portés dans le tableau l et la figure 2 . En lac, dans $81 \%$ des cas, les espèces se sont acclimatées. En revanche, elles ne se sont naturalisées que dans $9 \%$ des cas. On soulignera que les populations d'ombles de fontaine naturalisées sont très souvent de faible effectif, que ce soit en ruisseau ou en lac.

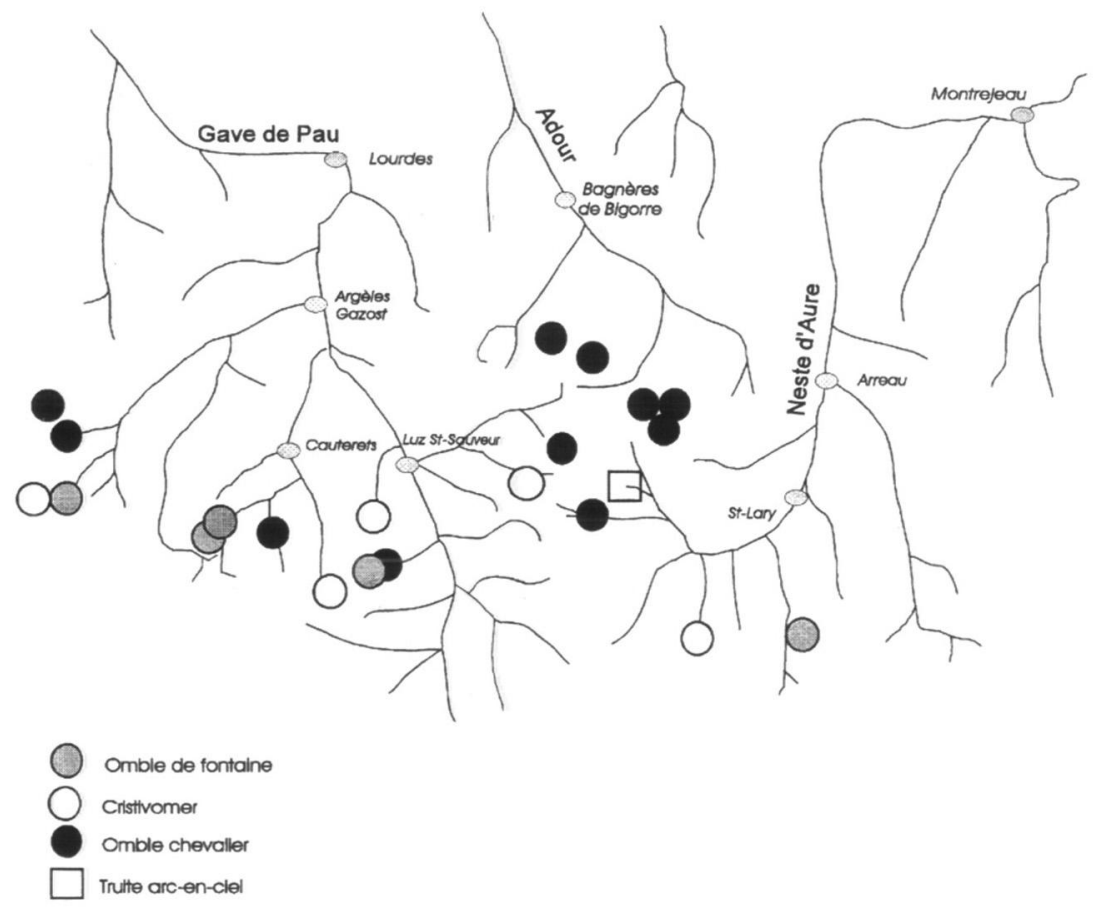

Figure 2

Populations naturalisées de Salmonidés.

Figure 2

Reproducing populations of Salmonids. 


\section{Tableau I}

Caractéristiques des introductions par espèce de Salmonidés.

TRF : truite commune

OBL : omble chevalier

SDF : omble de fontaine

CRI : cristivomer

TAC : truite arc-en-ciel

SP : splake

\section{Table I}

Characteristics of introductions per Salmonid species.

TRF : brown trout

OBL : arctic charr

SDF : brook trout

CRI : lake trout

TAC : rainbow trout

SP : splake

\begin{tabular}{|c|c|c|c|c|c|c|c|}
\hline \multirow{3}{*}{ Espéce } & \multirow{3}{*}{$\begin{array}{c}\text { Date de lère } \\
\text { introduction }\end{array}$} & \multicolumn{4}{|c|}{ Lacs } & \multirow{2}{*}{\multicolumn{2}{|c|}{\begin{tabular}{|c|} 
Ruisseaux \\
Naturalisations
\end{tabular}}} \\
\hline & & \multirow[t]{2}{*}{ Introductions } & \multirow[t]{2}{*}{ Acclimatations } & \multicolumn{2}{|c|}{ Naturalisations } & & \\
\hline & & & & avec certitude & avec présomption & avec certitude & avec présomption \\
\hline TRF & $?$ & 243 & 236 & 22 & 12 & & \\
\hline SDF & 1936 & 125 & 124 & 3 & 2 & 2 & 2 \\
\hline TAC & 1906 & 68 & 6 & 1 & 1 & 1 & \\
\hline OBL & 1936 & 16 & 15 & 11 & 3 & & \\
\hline CRI & 1953 & 21 & 11 & 5 & 2 & & \\
\hline SP & 1992 & 4 & $?$ & $?$ & $?$ & & \\
\hline
\end{tabular}
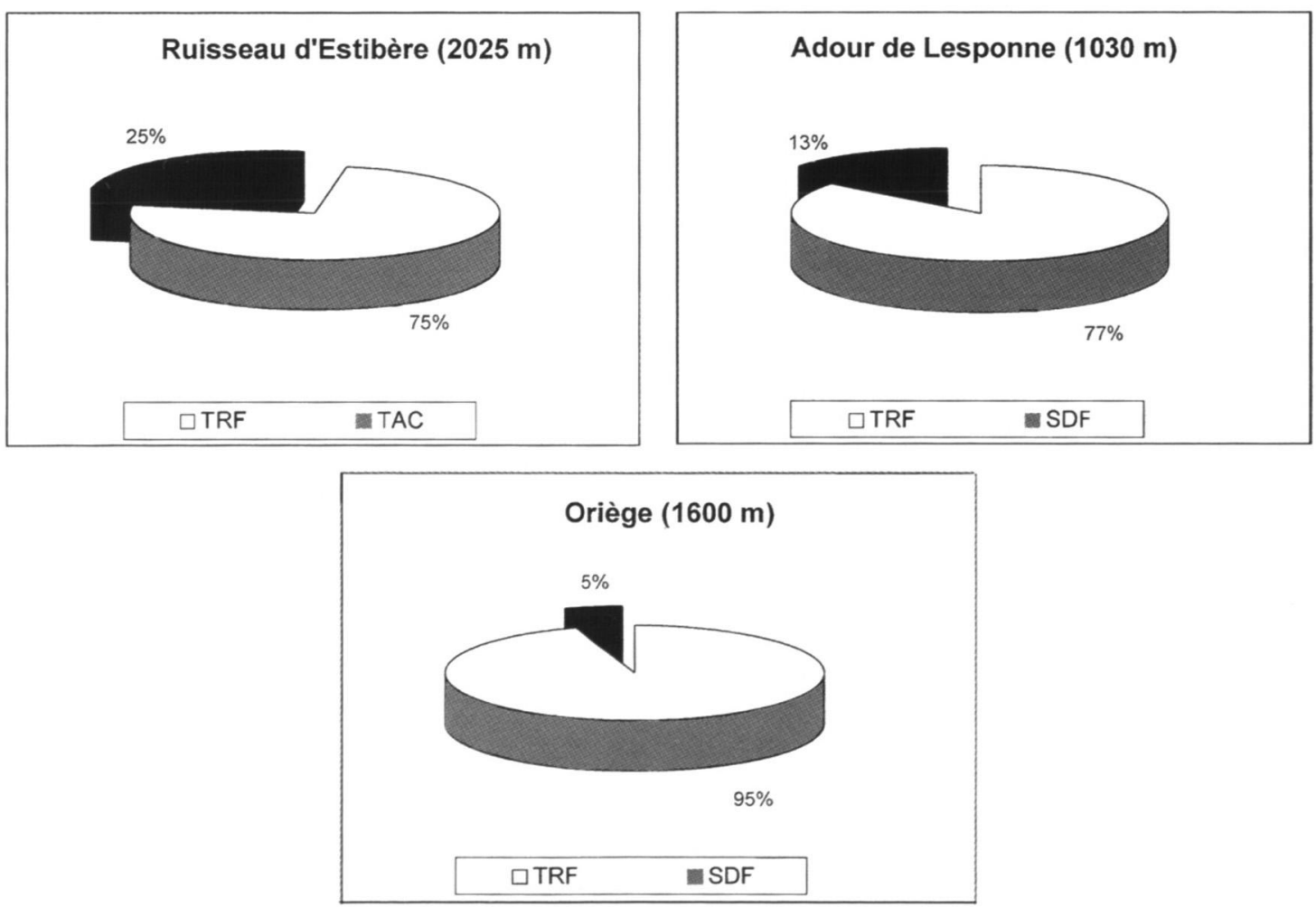

Figure 3

Comparaison des abondances de truites communes et de Salmonidés introduits dans 3 ruisseaux.

Figure 3

Comparison of brown trout and other introduced Salmonids abundances in 3 streams. 

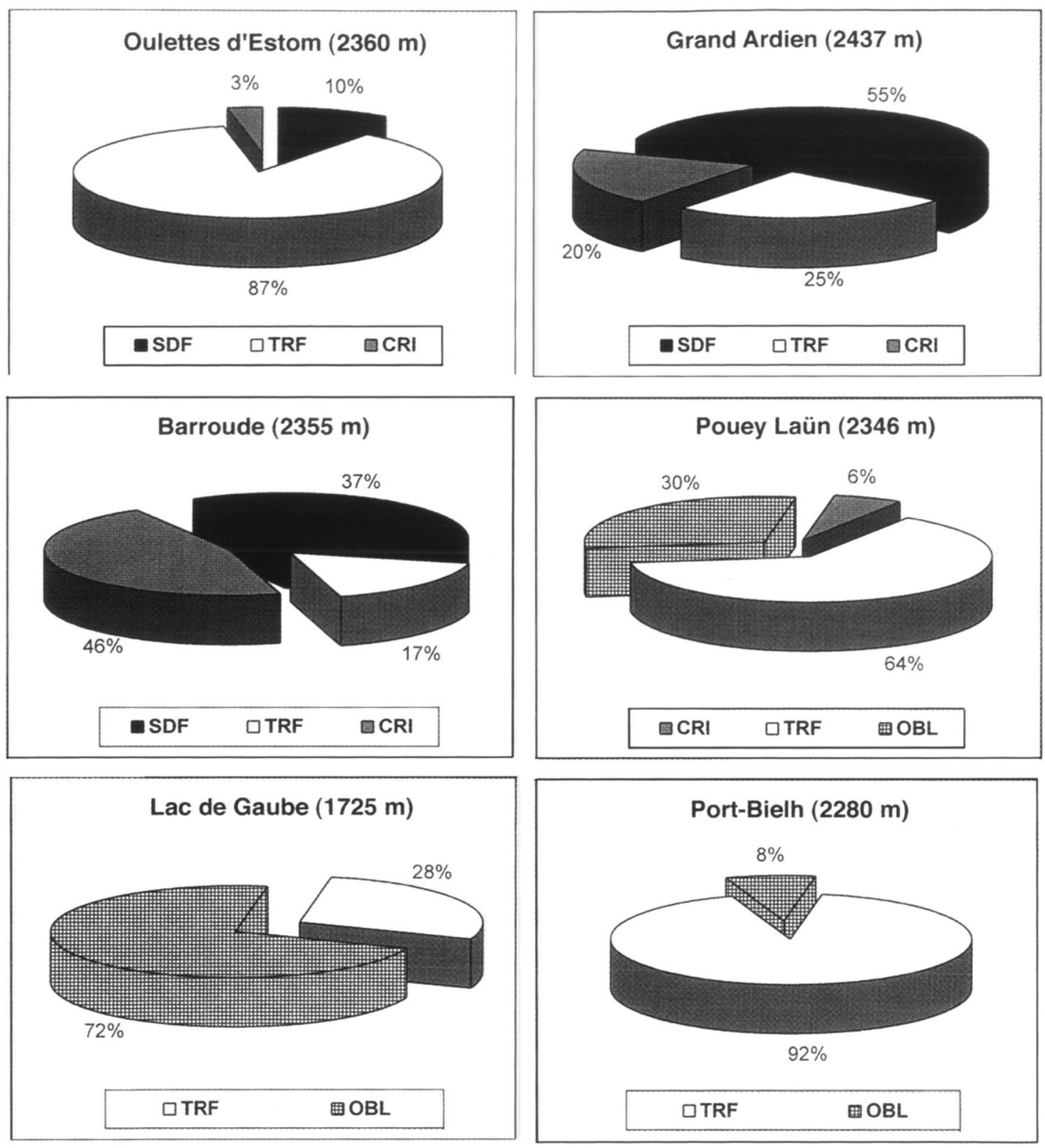

\section{Figure 4}

Comparaison des abondances de truites communes et de Salmonidés introduits dans 6 lacs.

\section{Figure 4}

Comparison of brown trout and other introduced Salmonids abundances in 6 lakes.

\section{Abondances}

En ruisseau, en situation de sympatrie, les abondances de truites communes (espèce indigène) sont systématiquement supérieures à celles des Salmonidés introduits (figure 3). En lac, en revanche, le peuplement n'est pas systématiquement dominé par la truite commune. Les proportions relatives des différentes espèces varient selon le lac considéré (figure 4). Celles-ci semblent dépendre des caractéristiques du lac, mais aussi et surtout des alevinages récemment réalisés. 


\section{Croissance}

Dans les deux ruisseaux étudiés, la croissance de la truite commune est plus lente que celle des espèces introduites (truite arc-en-ciel et omble de fontaine) (figure 5). En lac, la croissance de l'omble de fontaine est supérieure à celle des autres Salmonidés, à quelques exceptions près (figure 6). Dans le cas des lacs de haute altitude (>2300 m), l'omble de fontaine a une croissance toujours supérieure à celle de la truite commune. L'omble chevalier est quant à lui très souvent caractérisé par une croissance faible. Les études confirment l'importante longévité du cristivomer. Dans le lac glacé d'Estom Soubiran, des individus de plus de 13 ans ont été capturés. Les tailles variaient de 600 à $710 \mathrm{~mm}$. Ces individus s'étaient reproduits dès leur 8ème année $(420 \mathrm{~mm})$. Dans le lac glacé de Maniportet, la croissance du splake est inférieure à celle de l'omble de fontaine (figure 6). L'introduction ayant eu lieu en 1992, il est encore trop tôt pour se prononcer quant à sa naturalisation. Cette espèce, bien qu'hybride, possède en effet la possibilité de se reproduire (KRUEGER et MAY, 1991).
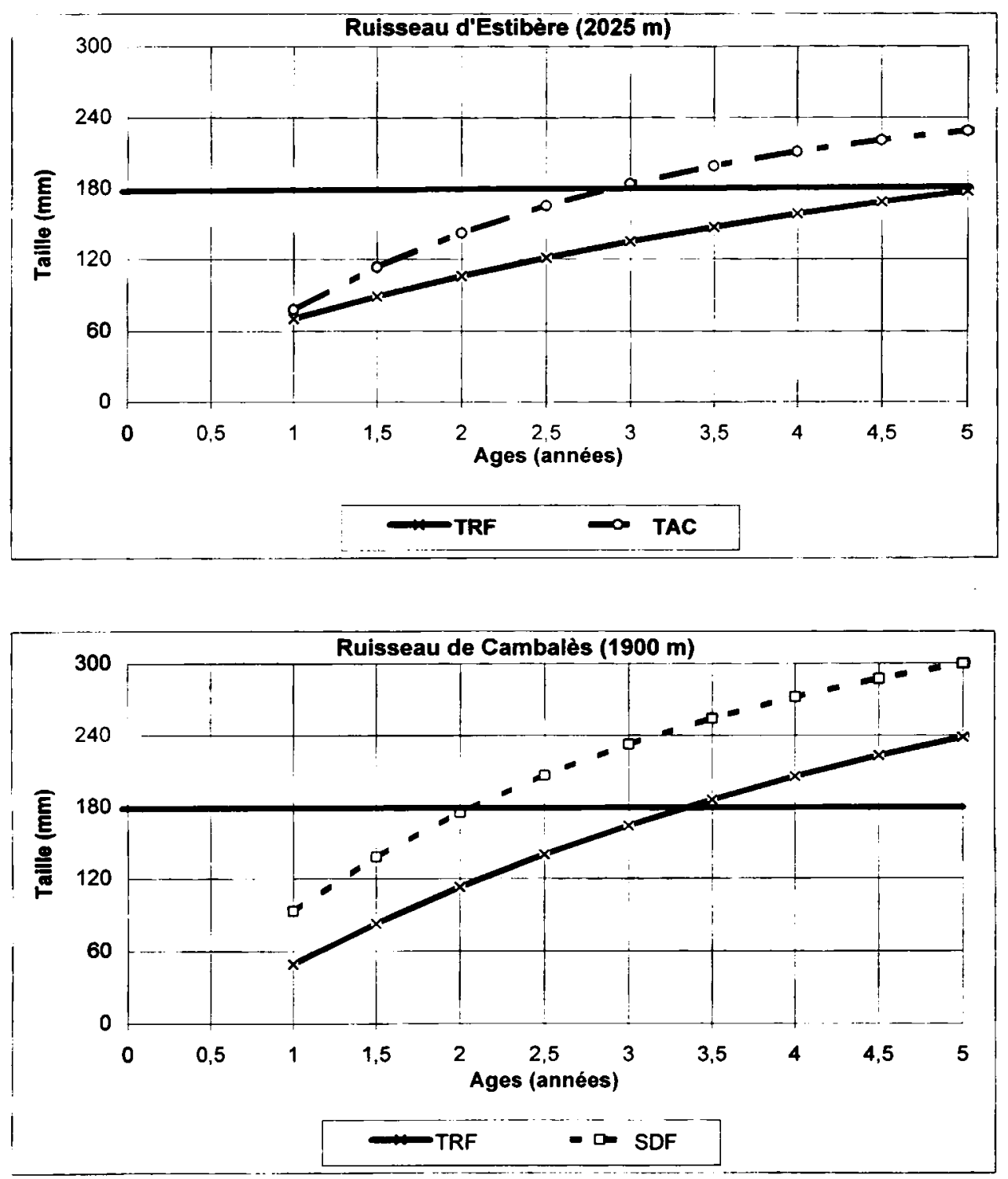

Figure 5

Comparaison des croissances de truites communes et de Salmonidés introduits dans 2 ruisseaux.

\section{Figure 5}

Comparison of brown trout and other introduced Salmonids growths in 2 streams. 

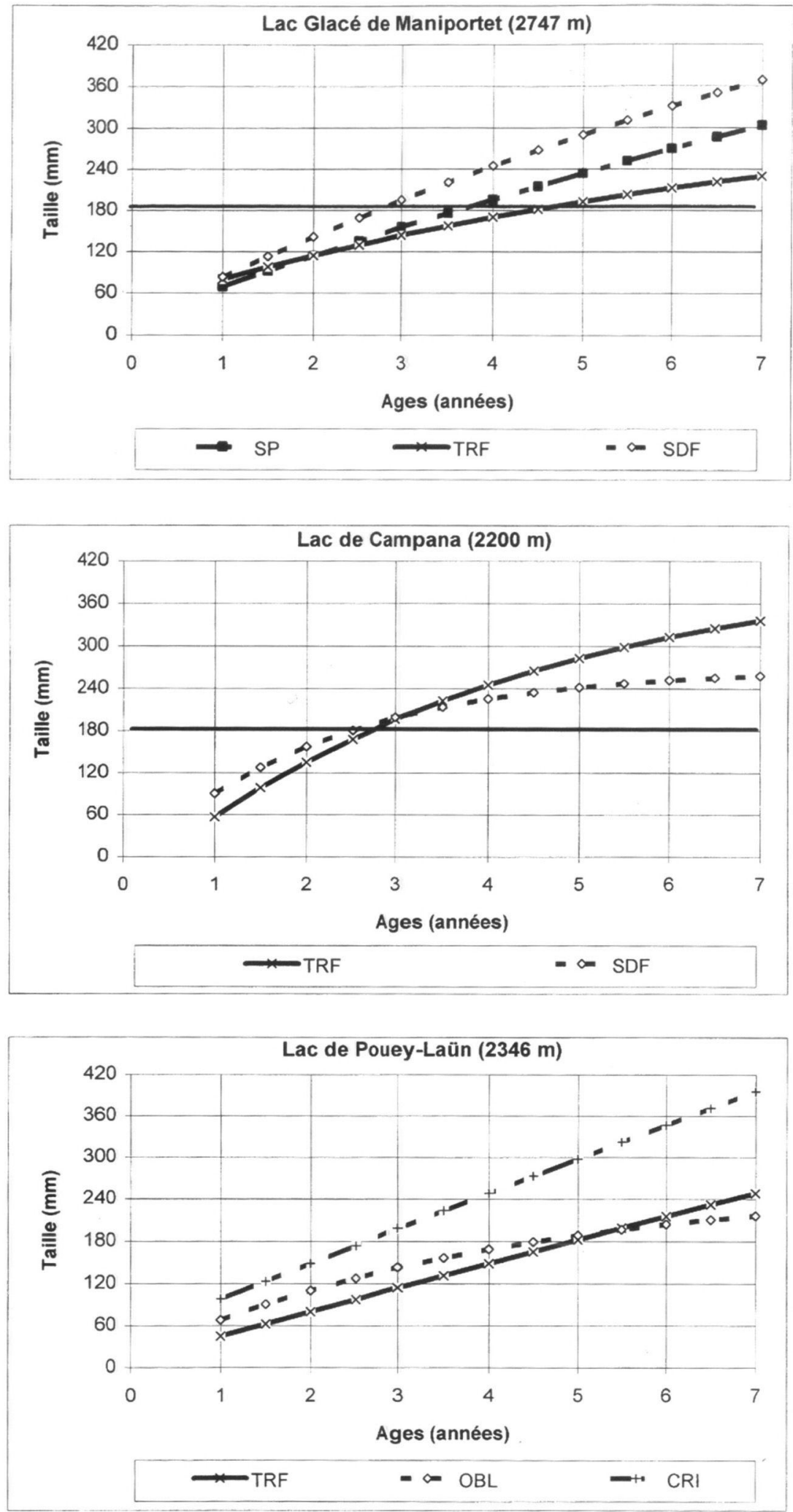

Figure 6

Comparaison des croissances de truites communes et de Salmonidés introduits dans 3 lacs.

Figure 6

Comparison of brown trout and other introduced Salmonids growths in 3 lakes. 


\section{Capturabilité par pêche à la ligne}

Dans le ruisseau d'Estibère, la capturabilité par pêche à la ligne de la truite commune est inférieure à celle de la truite arc-en-ciel (figure 7) $(65 \%$ des captures pour l'espèce introduite alors qu'elle ne représente que $25 \%$ des effectifs du peuplement). Dans les lacs d'Opale et de Costalade, on retrouve une situation identique avec l'omble de fontaine (figure 7).
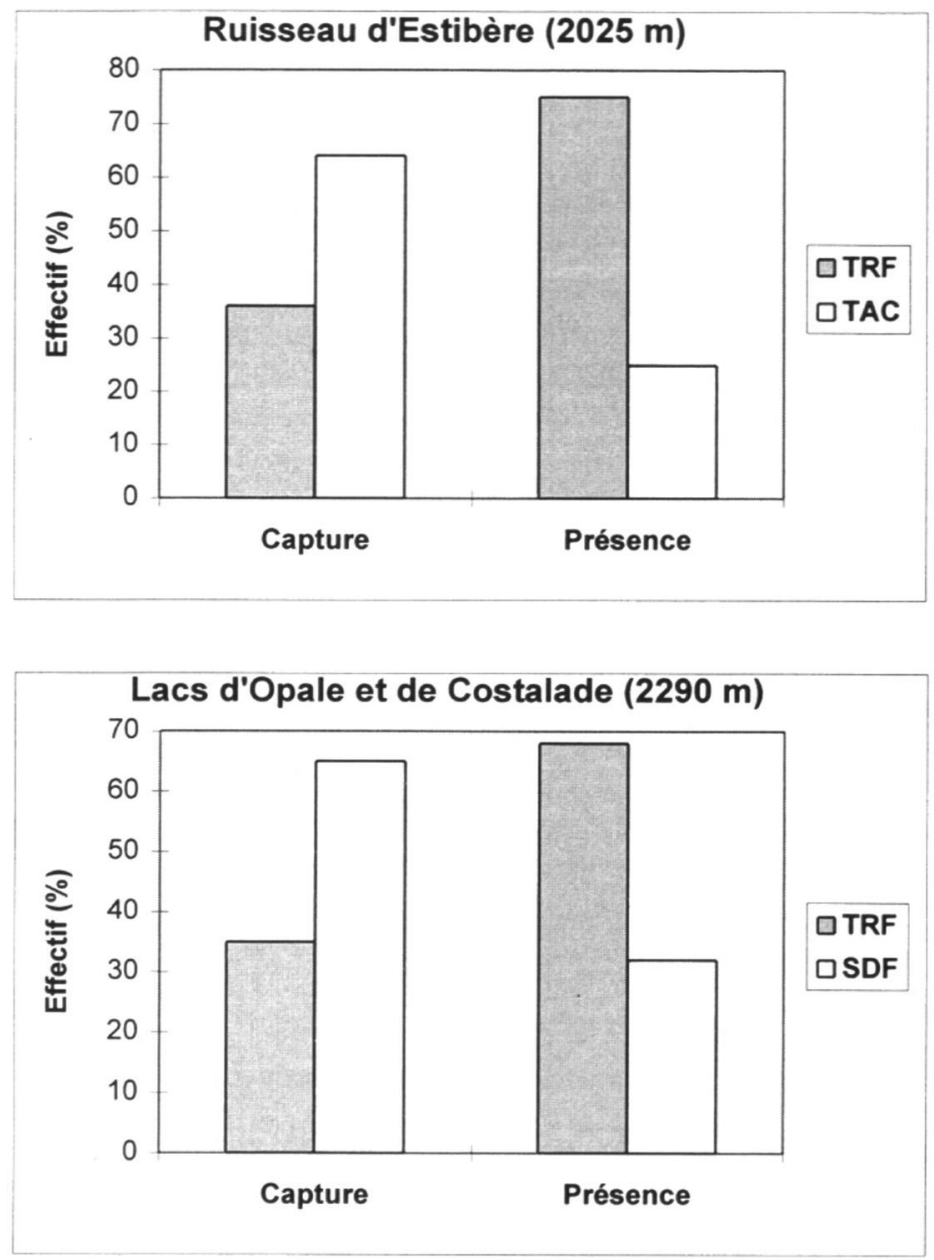

\section{Figure 7}

Comparaison de la capturabilité par pêche à la ligne de la truite commune et de Salmonidés introduits en lac et en ruisseau.

\section{Figure 7}

Comparison of brown trout and other introduced Salmonids angling capturability in lake and stream.

\section{DISCUSSION}

\section{Acclimatation et naturalisation}

Comme dans de nombreuses autres régions, les introductions de Salmonidés en lacs et ruisseaux d'altitude des Pyrénées ont débouché sur des acclimatations ( $81 \%$ des cas). Ces différents milieux ont donc offert une niche spatiale et alimentaire vacante susceptible d'être occupée temporairement par une espèce de poisson, ce qui constitue une base indispensable à l'introduction d'une espèce (LI et MOYLE, 1981 ; HERBOLD et MOYLE, 1986). Ces milieux 
sont pourtant caractérisés par des conditions écologiques hivernales très rigoureuses (prise en glace de novembre à mai pour les lacs et les ruisseaux d'altitude comprise entre 2000 et 2300 m (UZABIAGA, 1978 ; LAVANDIER, 1979) et faible température, inférieure à $1^{\circ} \mathrm{C}$ de novembre à mars pour les torrents (LAVANDIER, 1979)). Ces conditions ne semblent toutefois pas avoir limité les possibilités d'acclimatation des différentes espèces. En effet, les Salmonidés peuvent supporter des conditions environnementales très difficiles (CALKINS, 1989).

En revanche, ces facteurs conditionnent les faibles croissances des poissons et notamment de la truite commune dans les lacs d'altitude supérieure à $2300 \mathrm{~m}(<170 \mathrm{~mm}$ à 3 ans). De même, dans les ruisseaux de haute altitude $(>2000 \mathrm{~m})$, la truite commune se développe beaucoup plus difficilement que l'omble de fontaine ou la truite arc-en-ciel (180 $\mathrm{mm}$ à 5 ans dans le ruisseau d'Estibère contre $235 \mathrm{~mm}$ pour la truite arc-en-ciel). En ce qui concerne les autres espèces, les croissances du cristivomer (entre $280 \mathrm{~mm}$ et $410 \mathrm{~mm}$ à 7 ans) sont assez semblables à celles observées sur les lacs alpins (425 mm à 7 ans) (MARTINOT, 1979) ou canadiens (entre 359 et $542 \mathrm{~mm}$ à 7 ans) (SCOTT et CROSSMAN, 1974). De même, pour l'omble de fontaine, on retrouve des croissances (entre 168 et 248 $\mathrm{mm}$ à 3 ans) assez semblables à celles des populations nord-américaines (entre 148 et 270 $\mathrm{mm}$ ) (SCOTT et CROSSMAN, 1974). L'omble chevalier présente quant à lui des croissances faibles par rapport à celles enregistrées dans d'autres régions $(216 \mathrm{~mm}$ à 7 ans dans le lac de Pouey-Laün) (MACHINO, 1991). Dans certains lacs, comme le lac de Gaube, des phénomènes de nanisme apparaissent (PLASSEREAU, 1987) comme cela avait été observé en d'autres endroits (DUSSART, 1954 ; PECHLANER, 1984). En ce qui concerne le splake, contrairement aux données bibliographiques (SCOTT et CROSSMAN, 1974), sa croissance n'est pas supérieure à celle de l'un des parents, en l'occurrence l'omble de fontaine.

A l'inverse des acclimatations (156 cas sur 234 sites d'introductions de poissons non indigènes), les phénomènes de naturalisation sont beaucoup moins fréquents ( 20 cas). II est toutefois important de rappeler que les introductions ont été motivées par des objectifs halieutiques. En cela, les gestionnaires ont essentiellement évalué les potentialités d'acclimatation offertes à une espèce par un site, plutôt que les potentialités de naturalisation. Celle-ci dépend en premier lieu de l'espèce considérée. Dans les lacs, les acclimatations d'omble chevalier ont abouti dans $73 \%$ des cas à des naturalisations, et dans $45 \%$ des cas pour le cristivomer. En revanche, pour les autres espèces, en milieu lacustre, les cas de naturalisation sont beaucoup plus rares (moins de $10 \%$ pour la truite arc-en-ciel, l'omble de fontaine et la truite commune). Ce sont les exigences vis-à-vis des conditions d'habitat physique de reproduction qui expliquent ces différences. Les six espèces introduites sont toutes lithophiles (BALON, 1975), c'est-à-dire qu'elles enfouissent leurs oeufs dans le substrat. Mais au travers de ce trait comportemental commun, des différences existent. Le cristivomer, par exemple, dépose directement ses oeufs dans la cuvette lacustre dans les interstices des galets et rochers et sans creuser de nid (BALON, 1975). L'omble chevalier a un comportement similaire. Les oeufs sont déposés à des profondeurs supérieures à 20 ou $30 \mathrm{~m}$, sur un substrat grossier avec des écoulements sous-lacustres (DUSSART, 1954). A l'opposé, la truite commune, la truite arc-en-ciel et l'omble de fontaine construisent un nid dans une zone de courant. Des études de microhabitats ont clairement montré que les nids de truites communes étaient construits sur des sites où la vitesse variait de 10 à $45 \mathrm{~cm} / \mathrm{s}$ (DELACOSTE et al., 1995). L'omble de fontaine possède des exigences très proches de celles de la truite commune. Ces conditions de vitesse de courant ne se rencontrent que très exceptionnellement dans les lacs. La présence d'un émissaire ou d'un tributaire est donc indispensable à ces deux espèces pour effectuer leur reproduction. Cette condition n'est pas remplie dans la majorité des lacs pyrénéens, ce qui explique leur faible taux de naturalisation $(<10 \%)$ comparativement aux espèces typiquement lacustres. La plasticité des exigences en termes d'habitat et de nutrition permet aux Salmonidés de s'acclimater tant en milieu lacustre qu'en milieu lotique. En revanche, cette plasticité est fortement réduite au niveau de l'habitat de reproduction et conditionne directement les potentialités de naturalisation. 
Pourtant, les exigences en termes d'habitat de reproduction ne sont pas les seuls facteurs expliquant la naturalisation d'une espèce introduite. En effet, en ruisseau, les naturalisations d'ombles de fontaine et de truites arc-en-ciel sont peu nombreuses (seulement 3 cas recensés avec certitude), bien que les conditions de reproduction soient favorables. Trois éléments peuvent expliquer cette situation.

Tout d'abord, les conditions environnementales lors du développement intragravellaire des oeufs et des larves. La prise en glace ou les faibles températures peuvent entraîner d'importantes mortalités et de fortes fluctuations des effectifs (REISER et WESCHE, 1979 ; WALSH et CALKINS, 1986 ; CALKINS, 1989).

La compétition avec la truite commune constitue le deuxième élément à prendre en compte. En effet, cette espèce est considérée comme la plus agressive des espèces de Salmonidés et se place souvent en situation de dominance (KALLEBERG, 1958 ; NYMAN, 1970 ; FAUSCH et WHITE, 1981 ; GATZ et al., 1987). Dans le ruisseau d'Estibère, la compétition interspécifique joue un rôle important dans l'utilisation de l'habitat physique par la truite arc-enciel et la régulation des effectifs de cette espèce (BARAN et al., 1995a). Aux États-Unis, l'introduction de truites communes a abouti à de fortes réductions des effectifs de truites fardées et d'ombles de fontaine (WATERS, 1983 ; GRIFFITH, 1988 ; KRUEGER et MAY, 1991). Dans une rivière du Minnesota, la compétition, les fluctuations de l'environnement et la pêche ont conduit au remplacement progressif de la population d'ombles de fontaine (100\% des effectifs en 1965) par la truite commune (70 \% des effectifs en 1980) (WATERS, 1983). En ruisseau, la défense du territoire joue un rôle clé dans la régulation des densités (CHAPMAN, 1966). Des changements de niche spatiale sont souvent observés lorsque deux espèces de Salmonidés coexistent (GATZ et al., 1987). Toutefois, en lac, le comportement territorial est beaucoup plus réduit, en raison principalement de l'absence de courant (GRIFFITH, 1988). La compétition pour la défense d'un microhabitat de nutrition est moins marquée qu'en ruisseau. Cette observation pourrait expliquer la coexistence, voire l'importance des effectifs d'ombles de fontaine par rapport à la truite commune dans certains lacs des Pyrénées, notamment les lacs de haute altitude.

Le troisième élément est relatif à la capturabilité des différentes espèces par la pêche à la ligne. En effet, les capturabilités de la truite commune, de la truite arc-en-ciel et de l'omble de fontaine diffèrent nettement. Les deux dernières espèces possèdent des capturabilités supérieures à celle de l'espèce indigène (dans le ruisseau d'Estibère : 13 poissons/heure/pêcheur pour la truite arc-en-ciel contre 7 poissons/heure/pêcheur pour la truite commune). Ces résultats confirment ceux obtenus par d'autres auteurs (COOPER, 1952 in WATERS, 1983 ; NYMAN, 1970 ; MARSHALL et MCCRIMMON, 1970). WATERS (1983) considère que la pression de pêche et la compétition avec la truite commune expliquent une part importante des réductions d'effectifs d'ombles de fontaine dans les rivières du Minnesota. DUMAS (1976) relie certaines réductions d'abondances de truites arc-en-ciel dans le ruisseau d'Estibère aux effets de la pêche à la ligne. L'halieutisme aura d'autant plus d'effets que la taille légale de capture (180 mm dans le département des Hautes-Pyrénées) correspond bien souvent pour l'omble de fontaine à la taille atteinte au cours de la deuxième année, c'est-à-dire avant la maturation sexuelle des femelles. Cette espèce, comme la truite arc-en-ciel, est donc pénalisée par une taille légale de capture mal adaptée, qui ne lui permet pas d'effectuer un cycle de reproduction.

\section{Impacts des introductions sur les biocénoses autochtones}

Le suivi de nombreuses populations de truites communes en aval des lacs et des ruisseaux d'altitude des Hautes-Pyrénées a montré la très faible incidence sur celles-ci des introductions d'autres espèces de Salmonidés. La dominance de l'espèce indigène limite les risques de compétition en sa défaveur, et donc les réductions d'effectifs. Au niveau génétique, si les croisements entre espèces sont rares (quelques cas de croisement truite commune $x$ omble de fontaine), les incidences des repeuplements en truite commune sur les populations indigènes ne semblent pas négligeables (BERREBI et BEAUDOU, 1991). 
En ce qui concerne les autres composantes des biocénoses aquatiques, et notamment les batraciens, aucune expérimentation sur les effets des introductions de Salmonidés n'a été réalisée. II semble que les amphibiens soient beaucoup plus rares dans les lacs et ruisseaux peuplés de Salmonidés que dans ceux vierges de poissons. Des observations ponctuelles (ruisseau d'Estibère) confirment les donnèes bibliographiques relatives à ce sujet (RESETARITS, 1995), à savoir une forte réduction voire l'absence de batraciens en présence de Salmonidés. Une introduction récente de truite commune dans le lac du Gourg de Rabas a conduit à une forte diminution des effectifs de crapaud accoucheur (Alytes obstetricans) (BESSON, données non publiées). L'euprocte ou urodèle (Euproctus asper Dugês), espèce endémique des Pyrénées, a vu ses effectifs et le nombre de stations dans lesquelles il était présent diminuer notablement avec l'introduction de Salmonidés (ANGELIER, com. pers.).

Au niveau des invertébrés benthiques, les lacs et ruisseaux d'altitude sont des milieux riches, avec notamment la présence d'espèces endémiques à la chaîne pyrénéenne. Dans le ruisseau d'Estaragne (2000 m d'altitude), LAVANDIER (1979) recense 21 endémiques sur un total de 200 espèces. La présence de Salmonidés induit des réductions d'abondances pour certaines espèces, particulièrement les espèces de grande taille (invertébrés prédateurs), au profit des espèces de petite taille (HARVEY, 1993). Toutefois, en ruisseau, la prédation exercée par les poissons ne semble pas conduire à des disparitions d'espèces, l'hétérogénéité du substrat étant mise à profit comme refuge (BECHARA et al., 1993).

\section{Bilan des introductions par rapport aux objectifs attendus}

L'objectif des introductions de Salmonidés en haute montagne est un développement du loisir pêche dans ces écosystèmes particulièrement préservés du développement anthropique. Dans la majorité des cas, le but fixé est atteint, puisque les espèces se sont acclimatées et que la pêche en montagne est de plus en plus pratiquée par les pêcheurs locaux, mais également par des personnes extérieures au département ou à la région. Elles participent donc en ce sens au développement économique de la région.

\section{CONCLUSION}

Le tableau II rassemble des éléments pour un schéma de gestion des introductions de Salmonidés dans les Pyrénées. II insiste sur la relation entre objectifs des introductions, conditions environnementales et biologiques préexistantes, risques pour les biocénoses en place et résultats attendus. Les introductions sont replacées selon deux objectifs de gestion : une gestion patrimoniale et une gestion halieutique.

Les éléments rassemblés dans ce bilan ne constituent qu'une base géographique partielle, qu'il apparaît indispensable d'étendre à l'ensemble de la chaîne pyrénéenne. En effet, les introductions de Salmonidés sont pratiquées dans tous les autres départements de la chaîne (Ariège, Aude, Pyrénées-Orientales, Pyrénées-Atlantiques, Haute-Garonne) et des cas de naturalisation d'espèces sont connus (truite arc-en-ciel dans le massif des Carlit et les lacs des Bouillouses, omble de fontaine en Ariège et dans l'Aude). Une synthèse plus complète constituerait une base à l'élaboration d'une politique globale et concertée de gestion des introductions de Salmonidés dans les Pyrénées.

\section{REMERCIEMENTS}

Les auteurs tiennent à remercier pour leur collaboration et leur soutien financier le Parc National des Pyrénées, les gardes de la Fédération des A.A.P.P.M.A. des Hautes-Pyrénées, M. Gérard UZABIAGA, garde-moniteur du Parc National ; MM. MARTINEZ et CABAT pour les carnets de pêche et les nombreux stagiaires de l'ENSAT ayant participé aux campagnes de terrain dans des conditions climatiques parfois rigoureuses. 
Tableau II

Confrontation entre politiques d'introduction, contexte écologique, gestion halieutique et gestion patrimoniale.

\section{Table II}

Confrontation between introduction policies, ecological situation, fishing management and patrimonial management.

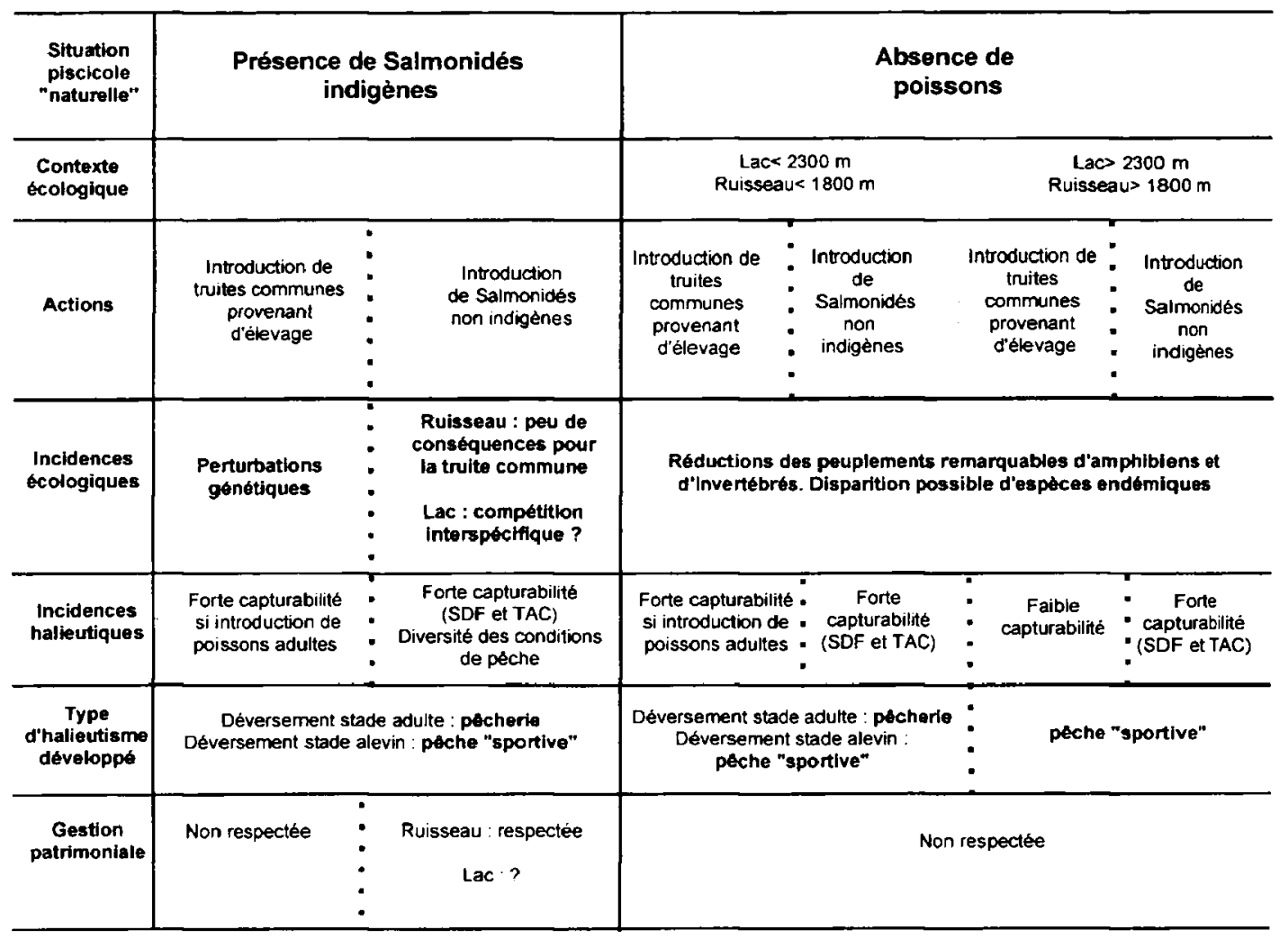

\section{BIBLIOGRAPHIE}

BAGLINIĖRE J.L., 1991. La truite commune (Salmo trutta L.), son origine, son aire de répartition, ses intérêts économique et scientifique. In BAGLINIÈRE J.L., MAISSE G., La truite, biologie et écologie, 12-22, INRA Publ., Paris.

BALON E.K., 1975. Reproductive guilds of fishes : a proposal definition. J. Fish. Res. Board Can., 32, 821-864.

BARAN P., 1995. Analyse de la variabilité des abondances de truites communes (Salmo trutta L.) dans les Pyrénées centrales françaises. Influence des échelles d'hétérogénéité d'habitat. Thèse de doctorat, Institut National Polytechnique de Toulouse, Laboratoire d'Ingénierie Agronomique, $147 \mathrm{p}$.

BARAN P., LASCAUX J.M., DELACOSTE M., DAUBA F., SEGURA G., 1995a. La compétition interspécifique entre la truite commune (Salmo trutta L.) et la truite arc-en-ciel (Oncorhynchus mykiss), influence sur la validité des modèles d'habitat. Bull. Fr. Pêche Piscic., 337-338-339, 283-290.

BARAN P., LASCAUX J.M., DELACOSTE M., ABAD A., 1995b. Etude des populations de truites communes (Salmo trutta L.) du ruisseau du Lac de Gaube. Rapport ENSAT, 19 p. 
BECHARA J.A., MOREAU G., HARE L., 1993. The impact of brook trout (Salvelinus fontinalis) on an experimental stream benthic community : the role of spatial and size refugia. J. Anim. Ecology, 62, 451-464.

BERREBI P., BEAUDOU D., 1991. La génétique des populations appliquée à la truite fario. Recueil des interventions et des conclusions, Journée Technique Internationale, Oronoz, Truite commune pyrénéenne : génétique et repeuplement, 5-19.

CALKINS D.J., 1989. Winter habitats of Altantic salmon, brook trout, brown trout and rainbow trout. A literature review. US Army Corps of Engineers, Special Reports, 89-34, 9 p.

CHAPMAN D.W., 1966. Food and space as regulators of salmonid populations in streams. The American Naturalist, 100 (913), 345-357.

CHIMITS P., 1960. Inventaire piscicole en 1960 des lacs de montagne des Basses et Hautes-Pyrénées. Bull. Fr. Piscic., 197, 136-148.

COOPER E.L., 1970. Management of trout streams. American Fisheries Society Special Publication, 7, 153-162.

CROWL T.A., TOWNSEND C.R., McINTOSH A.R., 1992. The impact of introduced brown and rainbow trout on native fish : the case of Australia. Reviews in Fish Biology and Fisheries, 2, 217-241.

DAUBA F., 1990. Etude du peuplement piscicole de 4 lacs des Pyrénées : le lac glacé d'Estom, le lac de Pouey-Laün, du Bastan inférieur et d'Estom Soubiran. Rapport ENSAT, 9 p.

DAUBA F., 1991. Etude des lacs du Grand Ardiden, du col d'Aratilles et d'Aratilles. Rapport ENSAT, $18 \mathrm{p}$.

DELACOSTE M., BARAN P., LASCAUX J.M., SEGURA G., BELAUD A., 1995. Capacite de la méthode des microhabitats à prédire l'habitat de reproduction de la truite commune. Bull. Fr. Pêche Piscic., 337-338-339, 345-353.

DUMAS J., 1976. Dynamique et sédentarité d'une population naturalisée de truite arc-en-ciel (Salmo gairdneri Richardson) dans un ruisseau de montagne, l'Estibère (Hautes-Pyrénées). Ann. Hydrobiol., 7 (2), 115-139.

DUSSART B., 1954. L'Omble-chevalier en France, Biométrie et Biologie. Ann. Stat. cent. Hydrobiol. appl., 5, 129-157.

ELLIOTT J.M., 1973. The food of brown and rainbow trout (Salmo trutta and Salmo gairdneri) in relation to the abundance of drifting of invertebrate in a mountain stream. Oecologia, 12, 329-347.

FAUSCH K.D., 1988. Tests of competition between native and introduced salmonids in streams. What have we learned? Can. J. Fish Aquat. Sci., 45, 2238-2246.

FAUSCH K.D., WHITE R.J., 1981. Competition between brook trout (Salvelinus fontinalis) and brown trout (Salmo trutta) in a Michigan stream. Can. J. Fish Aquat. Sci., 38, 1220-1227.

GATZ Jr, SALE A.J., LOAR J.M., 1987. Habitat shifts in rainbow trout : competitive influence of brown trout. Oecologia, 74, 7-19.

GRIFFITH J.S., 1988. A review of competition between cutthroat trout and other salmonids. In GRESSWELL R.E., Status and management of interior stocks of cutthroat trout, American Fisheries Society Symposium, 4, 134-140.

HARVEY B.C., 1993. Benthic assemblages in Utah headwater streams with and without trout. Can. J. Zool., 71, 896-900.

HERBOLD B., MOYLE P.B., 1986. Introduced species and vacant niches. The American Naturalist, 128, 5, 751-760.

KALLEBERG $H_{\text {., }}$ 1958. Observations in a stream tank of territoriality and competition in juvenile salmon and trout (Salmo trutta L. and Salmo gairdneri L.). Rep. Inst. Freshwater Res. Drottningholm, 39, 55-88. 
KRUEGER C.C., MAY B., 1991. Ecological and genetic effects of Salmonids introductions in North America. Can. J. Fish Aquat. Sci., 48 (suppl. 1), 66-77.

LAVANDIER P., 1979. Ecologie d'un torrent pyrénéen de haute montagne : l'Estaragne. Thèse de doctorat ès Sciences Naturelles, Université Paul Sabatier, $532 \mathrm{p}$.

LI H.W., MOYLE P.B., 1981. Ecological analysis of species introductions into aquatic systems. Trans. Am. Fish. Soc., 110, 772-782.

MACHINO Y., 1991. Répartition géographique de l'Omble chevalier (Poisson, Salmonidae, Salvelinus alpinus) en France. Diplôme Supérieur de Recherche, Université Joseph Fourier, Grenoble, $438 \mathrm{p}$.

MARSHALL T.L., McCRIMMON H.R., 1970. Exploitation of self-sustaining Ontario stream population of brown trout (Salmo trutta) and brook trout (Salvelinus fontinalis). J. Fish. Res. Board Can., 27, 1087-1102.

MARTINOT J.P., 1979. Ecologie et gestion piscicole des lacs de haute altitude du Parc National de la Vanoise. Thèse de 3ème cycle, Université Joseph Fourier, Grenoble, $128 \mathrm{p}$.

MASSAS C., 1859. Le pêcheur à la mouche artificielle et le pêcheur à toutes les lignes. 2ème éd., Garnier, Paris.

MCCRIMMON H.R., 1971. World distribution of rainbow trout (Salmo gairdneri). J. Fish. Res. Board Can., 28, 663-704.

McDOWALL R.M., 1984. Exotic fishes : the New Zealand experience. In COURTENAY W.R., STAUFFER J.R., Distribution, biology and management of exotic fishes, 200-214, Johns Hopkins University Press, Baltimore, MD.

MOREAU A., 1863. Pau, Eaux Bonnes, Eaux Chaudes. Fontaines 3ème éd., Paris.

MOYLE P.B., 1976. Inland fishes of California. University of California Press, Berkeley, CA, $405 \mathrm{p}$.

NYMAN O.L., 1970. Ecological interaction of brown trout Salmo trutta L., and brook trout Salvelinus fontinalis (Mitchill), in a stream. Can. Field-Natur., 84, 343-350.

PECHLANER R., 1984. Dwarf populations of arctic charr in high-mountain lakes of the Alps resulting from under-exploitation. In JOHNSON L., BURNS B.L., Biology of arctic charr, 319-327, Proceedings of the International Symposium on Arctic Charr, University of Manitoba Press, Winnipeg, MA.

PLASSEREAU O., 1987. Etude des populations d'omble chevalier (Salvelinus alpinus L.) des lacs de Gaube, Oncet et Cap de Long. Rapport ENSAT, $22 \mathrm{p}$.

PLASSEREAU O., 1989. Etude des populations de truite commune (Salmo trutta L.) dans trois lacs des Hautes-Pyrénées : le Peyregnet des Cambalès, l'Estagnol d'Escoubous, le Bastan inférieur. Rapport ENSAT, $18 \mathrm{p}$.

REISER D.W., WESCHE T.A., 1979. In situ freezing as a cause of mortality in brown trout eggs. Prog. Fish. Cult., 41, 2, 58-63.

RESETARITS W.J., 1995. Competitive asymmetry and coexistence in size-structured populations of brook trout and spring salamanders. Oikos, 73, 188-198.

SCOTT W.B., CROSSMAN E.J., 1974. Poissons d'eau douce du Canada. Min. Env. Canada, Ottawa, $1026 \mathrm{p}$.

UZABIAGA G., 1978. Morphométrie, physico-chimie et phytoplancton de trois lacs de barrage d'altitude des Pyrénées. Thèse de doctorat, Université Paul Sabatier, Toulouse, $111 \mathrm{p}$.

WALSH M., CALKINS D.J., 1986. River ice and salmonids. 4th Workshop on Hydraulics of River Ice, Montréal, Québec, Canada, D4.1-D4.26.

WATERS T.F., 1983. Replacement of brook trout by brown trout over 15 years in a Minnesota stream : production and abundance. Trans. Am. Fish. Soc., 112, 137-146. 